\title{
Estudio de caso: Intervención a una persona con dificultades en el lenguaje a nivel preescolar
}

\author{
Case study: Intervention for a person with language difficulties \\ at the preschool level
}

\author{
Palmenia Ignacia Pinochet Quiroz \\ Magíster en Educación Especial y Psicopedagogía, \\ Universidad Católica del Maule. \\ Coordinadora Técnico Pedagógico del Centro de Apoyo \\ a la Docencia y Aprendizaje, CADA \\ Universidad Católica del Maule, Talca, Chile \\ ppinochetq@ucm.cl
}

\section{RESUMEN}

El lenguaje es considerado uno de los procesos cognitivos complejos que requiere un desarrollo oportuno y una estimulación temprana debido a que sienta las bases para los aprendizajes y es determinante en las habilidades comunicativas de los niños. El presente estudio de caso da a conocer el proceso de evaluación e intervención de una menor de 4 años diagnosticada con TEL-Mixto con un alto nivel de afectación. Se describe la terapia fonoaudiológica y psicopedagógica, basada en estrategias de intervención mixtas. Los resultados de las 24 sesiones muestran avances positivos a nivel de lenguaje y autonomía en un período breve de tiempo. Este caso permite identificar la relevancia de una actuación oportuna y colaborativa ante la presencia de dificultades del lenguaje. Se discuten los resultados e implicancias para la práctica.

Palabras clave: lenguaje, trastornos del lenguaje, intervención, familia. 


\begin{abstract}
Language is considered a complex cognitive process that requires timely development and early stimulation because it is a foundational factor for learning and is crucial in the communication skills of children. The present case study describes the assessment and intervention process of a four yearsold child who had a diagnosis of Specific Language Impairment (SLI) that affected significantly her language skills. The intervention process included speech and language treatment as well as academic support. Mixed Strategies were included in the intervention plan. Despite the intervention was implemented in a short time (24 sessions), results showed positive outcomes both in language development and autonomy. This case allows identifying the relevance of a timely and collaborative strategy in the presence of language difficulties. Findings and implications for practice are discussed.
\end{abstract}

Key words: Language, Language Difficulties, Intervention, Family.

\title{
1. Introducción
}

El lenguaje es un código, un sistema de símbolos que permite a las personas expresar ideas y comunicarse, "es una habilidad social que promueve la interacción y también es una habilidad cognitiva que permite comprender, indagar y preguntar acerca de uno mismo y el mundo" (Allen \& Hart citado en Allen \& Cowdery, 2015, p. 415). En un sentido más amplio, el lenguaje es el sistema que regula gran parte de nuestras conductas y emociones, y le da una organización al pensamiento (Ardila, 2006).

Es imprescindible que el desarrollo de un(a) niño(a) siga las etapas e hitos "esperados" para su edad. Sin embargo, durante este proceso de adquisición también existen una serie de factores que inciden en el logro de los hitos, que pueden dar origen a trastornos del lenguaje. Estosson definidos como aquellas dificultades persistentes en la adquisición y uso del lenguaje en todas sus modalidades, debido a deficiencias de la comprensión o a la producción que incluye un vocabulario reducido, estructura gramatical limitada y deterioro del discurso, que están notablemente por debajo de lo esperado para la edad y cuyos síntomas se producen en las primeras fases del período de desarrollo. Estas dificultades no están asociadas a daño neurológico o cualquier otra condición médica (American Psychiatric Association [APA], 2013). 
Para el diagnóstico se deben considerar las características descritas, actualmente, en el Manual Diagnóstico y Estadístico de los Trastornos Mentales DSM - V (APA, 2013), en el cual la terminología de "Trastorno mixto del lenguaje y trastorno del lenguaje expresivo" es modificada por "Trastorno del lenguaje", compartiendo las características mencionadas. Para algunos niños y niñas los procesos en la adquisición del lenguaje son lentos y difíciles y cerca de un 5 a 10\% de los niños que inicia con su formación pre-escolar manifiesta estas alteraciones. En consecuencia, éste es uno de los cuadros más frecuentes de problemas en la comunicación oral en la población infantil, sin embargo, los niños con este tipo de dificultades no evidencian pérdida auditiva, daño cerebral, desempeños cognitivos descendidos ni déficit motor (Levit, 2004; Acosta, 2012).

Las dificultades asociadas a tales entidades diagnósticas inciden en el proceso de desarrollo del lenguaje y, en consecuencia, en la adquisición de nuevos aprendizajes. Por tanto, una atención temprana e intervención oportuna en esta área permitirá obtener avances y mayores desempeños lingüísticos en función de las características del paciente, dado que, los estudiantes que ingresan por diagnóstico de TEL Mixto o Expresivo' a los Programas de Integración Escolar (PIE) en Chile representan el 18\%, constituyendo el tercer diagnóstico más frecuente de todos los niños integrados (Fundación Chile y Centro de Estudios MINEDUC, 2013).

En este caso, específicamente, se abordaron sintomatologías relacionadas con el Trastorno Específico del Lenguaje (en adelante TEL), el cual será entendido a partir del Decreto $N^{\circ} 170$ como una limitación significativa en el nivel de desarrollo del lenguaje oral, que no se explica por una causa aparente: déficit sensorial, auditivo o motor, por discapacidad intelectual, trastornos psicopatológicos, entre otros (Ministerio de Educación, 2009). En la intervención del lenguaje de niños con TEL se implementan, generalmente, modelos de intervención naturalista (Acosta y Moreno, 2005), Ios cuales tienen como objetivo lograr una comunicación exitosa entre el niño y todos sus interlocutores que actúan en una situación lingüística y comunicativa, beneficiando aspectos formales del lenguaje.

1 En el estudio se utilizará la terminología TEL (Trastorno Específico del Lenguaje) debido al diagnóstico inicial presentado, sin embargo, dada la denominación actual el concepto "TEL" hace referencia al Trastorno del Lenguaje (APA, 2013). 
Este enfoque tiene lugar en situaciones naturales y comunes para el niño, y otorga un papel preponderante a la participación de las personas que conviven normalmente y durante mucho tiempo con los niños (como los padres, cuidadores y maestros). Es por esto que, además, se consideran estrategias del Modelo Centrado en la Familia (Dunst et al., citado en GarcíaSánchez, et al. y Hernández-Pérez, 2014) y el Modelo Ecológico Sistémico (Burger, 2014). Bajo estos modelos, la familia es el componente estimulador y facilitador de oportunidades de aprendizaje por excelencia, teniendo una participación activa en los procesos de atención e intervención de su hijo(a), así como también un trabajo colaborativo con los profesionales que participan en el proceso de enseñanza y aprendizaje del menor.

En consecuencia, se presenta el estudio de caso de una estudiante preescolar con diagnóstico inicial de TEL-Mixto y con antecedentes de factores de riesgo asociados, a quien se le realizó un proceso de intervención que involucró estrategias de modelos mixtos de estimulación del lenguaje, con el propósito de aumentar su desempeño a nivel comunicativo y social.

\section{Método}

\section{Datos personales del paciente}

El caso se basó en una niña de 4 años con 8 meses, a quien llamaremos Jo. Ella inició sus estudios en un establecimiento educativo particular subvencionado en el nivel de prekinder con diagnóstico fonoaudiológico previo de "Trastorno específico del lenguaje de tipo mixto" emitido por fonoaudióloga particular. La menor pertenece a una familia nuclear, vive con ambos padres y una hermana mayor de 7 años.

Ante la presencia de un déficit significativo a nivel de lenguaje expresivo y receptivo que influía en su desempeño académico, observado por la educadora del nivel, surgió la necesidad de recabar información adicional de tipo anamnésica y aplicar evaluaciones tanto fonoaudiológicas como psicopedagógicas por el equipo de profesionales del Programa de Integración Escolar (en adelante PIE), integrado por Fonoaudióloga y Profesora de Educación Especial para complementar el diagnóstico inicial e identificar los apoyos que requeriría la menor para fortalecer su desempeño a nivel educativo. 


\section{Referencia y objetivos}

En relación a los antecedentes recopilados en el caso, se toma como referencia el proceso de evaluación diagnóstica propuesto por el Decreto Nº 170 (MINEDUC, 2009) debido a que es la normativa que entrega los lineamientos mínimos para la evaluación de estudiantes con necesidades educativas especiales (NEE) pertenecientes a establecimientos educacionales con PIE.

Las características presentes en la niña, en el área del lenguaje evidencian una comunicación preferentemente gestual, producción ininteligible, relato de experiencias disminuido, alteración de la comprensión de conceptos abstractos y relatos, lenguaje ecolálico y dificultad para seguir instrucciones de diversa complejidad. La afectación del lenguaje y los componentes descritos fueron considerados como base para el proceso de evaluación y posterior plan de intervención.

El objetivo del plan de acción fue intervenir los componentes del lenguaje descendidos a partir de modelos mixtos de estimulación en una alumna con diagnóstico de TEL-Mixto y con antecedentes de factores de riesgo asociados.

\section{Proceso evaluativo}

El equipo profesional del Programa de Integración Escolar realizó un proceso de evaluación adicional a Jo, el cual consistió en obtener información basada en el modelo descriptivo evolutivo (Acosta y Moreno, 1999), específicamente, comparando su rendimiento frente a criterios de "hitos" esperados para su edad y técnicas de recogida y análisis de información en procesos evaluativos, por medio de la aplicación de una anamnesis/entrevista a la familia.

A partir de la información anamnésica recogida, se identifica que Jo durante su desarrollo fue diagnosticada con macrocefalia por el médico pediatra, la cual no fue intervenida en tal período. Por lo tanto, se desconoce su nivel de daño o afectación en el sistema nervioso central. En esta etapa, la alumna estuvo en terapia ocupacional por dos meses debido a sus dificultades motoras y con terapia fonoaudiológica, en esta última se estableció el diagnóstico de "Trastorno específico del lenguaje mixto".

Los antecedentes pre, peri y post natal muestran que el parto fue por cesárea debido a que la madre presentó complicaciones por placenta previa y desprendimiento, lo que provocó sangrado por tres horas esperando asistencia médica. Tras el parto no se observaron dificultades presentes en la menor. Al año con siete meses, Jo presentó varicela, fiebre alta y estatus 
convulsivo, manifestado por movimientos de las extremidades cada una hora durante dos días, por lo cual estuvo hospitalizada en dos ocasiones. A partir de lo que menciona la madre no tuvo asistencia médica oportuna. La niña presentó virus respiratorio sincicial y a los dos años con siete meses neumonía bacteriana. Jo no tiene las vacunas correspondientes a su nivel de desarrollo por decisión de los padres.

En relación al desarrollo motor, Jo presentó control cefálico a los seis meses y bipedestó al año con ocho meses, evidenciando caídas frecuentes e hipotonicidad muscular. En el área visual y auditiva no se observan dificultades aparentes.

En cuanto al desarrollo del lenguaje este evidencia una afectación de todos sus componentes: (a) a nivel fonológico, la menor hace uso de múltiples procesos de simplificación fonológica (PSF); a nivel de estructura silábica, asimilación y omisión de letras, presenta un habla ininteligible; (b) a nivel morfosintáctico, hay un retraso en la adquisición de determinadas categorías morfológicas y bajo promedio de longitud del enunciado; (c) a nivel semántico existe una incorporación lenta de palabras al vocabulario pasivo (comprensivo, almacenado en el sistema semántico) o productivo (palabras de uso cotidiano), dificultad para recordar el término con el que se denomina al referente y para establecer relaciones entre palabras que pertenecen a una misma categoría semántica; (d) a nivel pragmático escasa participación espontánea en situaciones de conversación grupal o colectiva, es una comunicadora pasiva, dificultad para iniciar y mantener turnos conversacionales, habilidades comunicativas y conversacionales limitadas (Acosta y Moreno, 2005).

En el proceso evaluativo integral no fue posible la aplicación de pruebas fonoaudiológicas estandarizadas, debido al nivel de afectación tanto expresiva como comprensiva del lenguaje. Por ende, se observaron rutinas, hábitos, fortalezas y debilidades en sala de clases, sala de recursos, y también, a partir de la información recabada de sus padres por medio de la anamnesis aplicada y actividades cotidianas.

En este proceso se identificó un déficit significativo a nivel de lenguaje y en aspectos relacionados con su autonomía (por ejemplo, en actividades de la vida diaria propias de su edad), con inseguridad para realizar las actividades diarias que demanda el entorno o que puede requerir o solicitar ella misma.

En base a los patrones lingüísticos (comunicativos) propuestos por Owens (2008), las características presentes en la niña se encuentran 
substancialmente por debajo de lo esperado para su edad cronológica, las cuales corresponden a un desarrollo de un menor de dos años de edad. A los 4 años debería disponer de un vocabulario amplio, enunciados de 3 a 4 palabras, pronombres personales, pronombres posesivos claros (para ti, para mí), comprender frases pasivas, reparar quiebres comunicativos, hablar de cosas presentes, iniciar temas, adaptarse a las necesidades comunicativas del oyente y lograr espontaneidad, desarrollo de aspecto básicos de toma de turnos, comprender relaciones causa-efecto, manejo de colores, comprensión de opuestos, distancia y témporo espaciales, resolver problemas simples, desarrollo de dífonos consonánticos y una eliminación progresiva de PSF.

Se aplicó el Test de Desarrollo Psicomotor TEPSI (Haeussler \& Marchant, 1985) tras un mes de intervención, para recoger información de las áreas de lenguaje, coordinación y motricidad. Los resultados obtenidos manifestaron retraso en la totalidad de la prueba. De manera específica, Jo presentó retraso en el área del lenguaje en las áreas expresivas y comprensivas, manejo de conceptos básicos, vocabulario y en la capacidad de describir y verbalizar. Para poder identificar conceptos o vocabulario, la menor necesita que sea presentado de forma concreta y real, sino este concepto "no existe en ese momento". A nivel de coordinación se encuentra en la categoría de normalidad en acciones grafomotrices y movimientos finos por medio de la manipulación de objetos y también en factores perceptivos y representacionales. En el área de motricidad se encuentra en el rango límite (categoría de riesgo), presenta control del cuerpo en un acto breve y sus mayores dificultades están asociadas al equilibrio.

\section{Integración de resultados}

En función a los antecedentes recopilados y resultados obtenidos de las evaluaciones desarrolladas a nivel fonoaudiológico y psicopedagógico, se descarta el diagnóstico de base inicial de TEL-Mixto presente en Jo, debido a que el Trastorno Específico del Lenguaje no se explica por causa aparente (MINEDUC, 2009). Por el contario, en este caso se identifican signos de alerta relacionados con alteraciones del neurodesarrollo, posiblemente producto de la fisiopatología que presentó la menor en los primeros años de vida y que ha impactado en desmedro del desarrollo típico a nivel psicomotor y de lenguaje. En consecuencia, surge la hipótesis de que Jo presenta un Trastorno del lenguaje asociado a alteraciones del neurodesarrollo. 


\section{Intervención}

Se realizó un proceso de intervención integral siguiendo los modelos mixtos de intervención, centrado en la familia y ecológico sistémico. Jo asistió a dos sesiones semanales de 45 minutos con fonoaudióloga en aula de recursos (16 sesiones totales) y se realizaron intervenciones una vez a la semana (de 1 hora y 30 minutos, 8 sesiones totales) en aula regular por la profesora de Educación Especial en el ámbito de comunicación (lenguaje expresivo y comprensivo) durante dos meses. La familia asistió dos veces al mes a sesión con fonoaudióloga y profesora de Educación Especial con la finalidad de orientar el trabajo realizado con la alumna y habilitar a los padres con estrategias específicas de apoyo en el hogar para generar mayores oportunidades de aprendizaje en su contexto cotidiano y de esta manera hacer más efectivas las intervenciones llevadas a cabo con la niña.

El plan de intervención se focalizó, principalmente, en trabajar habilidades de lenguaje y favorecer el aumento de la autonomía en la menor. A nivel de lenguaje expresivo y comprensivo se toma como referencia los hitos esperados para la edad cronológica de la menor según Owens (2008), para, a partir de estas características, nivelar las áreas descendidas. Se abordaron todos los componentes del lenguaje oral que se encontraban descendidos en la alumna: fonológico, morfosintáctico, semántico y pragmático, a través de estrategias de intervención propuestas por Monfort y Juárez (2004) y Acosta y Moreno (2005), y estrategias de intervención naturalistas que fueron aplicadas de manera combinada con las planteadas inicialmente. El rol de los padres en este proceso fue esencial para complementar el trabajo realizado en la escuela y fortalecerlo en el hogar, por ende, en las reuniones de trabajo se les capacitó en el uso del material y estrategias de estimulación. Tales materiales fueron creados en el establecimiento y se les facilitaron a los padres para su uso en el hogar.

De manera transversal se utilizó el andamiaje Vigotsky (1993), metodología empleada en base a la importancia de facilitar el lenguaje a través de la interacción activa del niño con un adulto preparado para proporcionarle ayuda, guía y apoyo, y feedback correctivo utilizado para tratar los errores gramaticales y de semántica.

A nivel de lenguaje expresivo, las estrategias empleadas consideraron: imitación directa, aumento de vocabulario pasivo, estructuración de frases simples, modelado del lenguaje, expansión y extensión (modelos de intervención naturalista), expansión morfosintáctica y extensión semántica. La imitación directa fue empleada por medio de juegos y canciones, la 
cual potencia la memoria auditiva, el sentido del ritmo y de la entonación, también favorece la articulación de fonemas y palabras, y la incorporación de estructuras sintácticas. La estructuración de frases fue abordada a través del apoyo de fichas y/o tarjetas para elaborar frases, principalmente la intención imperativa de solicitar: Yo.... quiero.... (cada palabra fue apoyada con una tarjeta o ficha).

Para fortalecer el lenguaje comprensivo, las estrategias consideraron: seguimiento de instrucciones, ejecución de órdenes simples (1 criterio) de manera concreta y en el contexto familiar y escolar, y el aumento de vocabulario (categorías semánticas: partes del cuerpo, frutas, animales, objetos), a través de material concreto (lo más real posible), mediante juegos de identificación y de roles, los cuales fueron enviados al hogar de la niña para su refuerzo.

La intención comunicativa fue trabajada a través de instancias provocadas de manera explícita, en situaciones cotidianas y en actividades escolares, con el propósito de instaurar rutina social, mantener contacto ocular, responder a preguntas, respetar turnos de habla y utilizar el lenguaje para: pedir ayuda, solicitar y jugar.

La autonomía fue abordada en actividades cotidianas, a través del empleo de acciones concretas y de ejecución diaria que son solicitadas a la niña y acorde a su edad, por ejemplo: abrir su mochila, guardar algún elemento, pedir y realizar acciones. Las actividades se realizaron rutinariamente, otorgando tiempos de práctica: primero partió con un acompañamiento y modelado de las órdenes a realizar. Luego, se fomentó su autonomía de manera paulatina.

\section{Resultados}

\section{Valoración de la intervención}

A partir de las estrategias empleadas en este caso, se evidenciaron cambios positivos a pesar de que el proceso de intervención comprendió 24 sesiones (2 meses) por medio de rutinas breves. Los resultados fueron obtenido a través de la aplicación de un instrumento evaluativo no estandarizado para evaluar de manera procesual los avances obtenidos por la alumna a partir de los componentes trabajados en el plan de intervención. Los principales resultados se presentan a continuación. 


\section{Lenguaje expresivo y comprensivo}

A nivel de forma, contenido y uso (Bloom, 1978), los resultados obtenido por Jo son: Instauración de la estructura "Yo, quiero ...." con ayuda de tarjetas y/o fichas que apoyan la estructuración de la solicitud. Se reconoce como primera persona "Sabe que ella es Jo", solicita ayuda cuando lo requiere, se ha realizado traspaso de vocabulario pasivo a activo aumentando la producción de palabras de alta frecuencia correspondientes a categorías semánticas básicas.

Jo fortaleció la intención comunicativa de rutina social en relación a saludar y despedirse cuando va a algún lugar. Expresa gustos y disgustos frente a alguna actividad, tarea u otro, y existe mayor expresión e intención comunicativa con sus pares y adultos. Sigue instrucciones simples con acciones de uso cotidiano y concretas, por ejemplo: abrir su mochila, guardar algún elemento, buscar un objeto y realizar una actividad escolar.

\section{Autonomía}

A nivel de autonomía, los avances obtenidos están relacionados a una mayor disposición al trabajo en diferentes contextos, identifica una estructura de trabajo frente a una actividad, sigue instrucciones de baja complejidad, realiza acciones y genera solicitudes simples, y ejecuta tareas concretas dadas por los adultos, utiliza su lenguaje para pedir ayuda, solicitar, relacionarse con los demás, preguntar y jugar.

La comparación de resultados entre el desempeño inicial y el obtenido en las intervenciones se presenta en las tablas I y II. 
Tabla 1

Comparación del desempeño inicial y actual

\section{Diagnóstico inicial}

Habla ininteligible

\section{Resultados tras intervención}

Persisten procesos de simplificación fonológica (PSF).

Expresa palabras simples, en sílaba directa de extensión trisilábica.

Discrimina por concepto: casa/taza.

Fonemas aún no desarrollados

No estructura frases

Dislalia evolutiva de /r/ y /r'/- /g/ y /ch/.

Estructura una frase simple de 3 palabras con apoyo visual y kinésico.

Inseguridad en la expresión del lenguaje y realización de acciones

Déficit en vocabulario

No realiza descripciones/ verbalizaciones de acciones.

Déficit en el manejo de conceptos básicos
Mayorseguridadal expresarycomunicarse con su entorno. Existe inseguridad en la niña en relación a las palabras que representan una dificultad en su emisión, por tanto evitar producirlas.

Vocabulario pasivo en proceso de activación: maneja vocabulario básico de uso cotidiano y de alta frecuencia relacionado a frutas, animales, elementos del hogar.

En proceso de desarrollo.
Requiere potenciar lenguaje lógico matemático.

Maneja locativos espaciales (arriba y abajo) y tamaño (grande y chico) sólo presentados de manera concreta. 
Tabla II (continuación)

Comparación del desempeño inicial y actual

\begin{tabular}{ll}
\hline \multicolumn{1}{c}{ Diagnóstico inicial } & \multicolumn{1}{c}{ Resultados tras intervención } \\
\hline Comunicadora pasiva & $\begin{array}{l}\text { Inicia conversaciones con adultos y su } \\
\text { entorno. }\end{array}$ \\
Responsiva & Pregunta sobre situaciones. \\
& Pide ayuda. \\
& Presenta sonrisa social. \\
No presenta rutina social & Saluda y se despide al llegar o irse de un \\
& Iugar. \\
Baja autonomía & Disposición al trabajo. \\
& Sigue y ejecuta instrucciones simples con \\
& apoyo (concreto y/o gráfico). \\
\hline
\end{tabular}

Estos resultados son presentados de manera inicial a partir de los dos meses de trabajo con la menor, sin embargo, el plan de intervención y los ajustes asociados a partir de los resultados son mantenidos durante el año escolar.

\section{Discusión y conclusiones}

El lenguaje es considerado uno de los procesos cognitivos complejos y sustento para el desarrollo de otros aprendizajes importantes en etapas educativas, como la lectura y la escritura. Sin embargo, también se ve afectado por el funcionamiento ejecutivo y procesos como la memoria y atención, que actúan de manera intrínseca en conjunto para el logro de los aprendizajes que, en este estudio de caso, se encontraban en déficit, dada las dificultades observadas en la menor.

Se vuelve imprescindible pesquisar de manera oportuna posibles riesgos de retraso y detectar las causas de origen, con la finalidad de contar con conocimiento de las características de base, detectar las necesidades, entregar herramientas y estrategias de estimulación temprana a las familias, acordes a las necesidades de quien las presente.

En este caso fue necesario haber otorgado apoyos ajustados y oportunos a los padres de la menor de manera temprana, con la finalidad de minimizar 
las dificultades presentes. A pesar de esto, la edad de la niña favoreció que las intervenciones fueran más efectivas, dado que se encuentra en una etapa donde el cerebro presenta plasticidad cerebral y los cambios logren ser más significativos al desarrollo de funciones psíquicas y la estructuración de la personalidad (Feldman, 2007; MINSAL, 2008; Puche et. al., 2009).

Se concluye que existió un déficit a nivel de detección temprana de riesgos en el desarrollo, ya que el diagnóstico dado a la menor fue tardío y no hubo procesos de evaluación pertinentes para pesquisar si los antecedentes sobre dificultades en el embarazo, parto y del neurodesarrollo provocaron alguna alteración en sus hitos evolutivos. Por lo tanto, no se entregaron apoyos de forma oportuna. Esto pone de manifiesto que existe una necesidad en el sistema de salud frente a los procesos de detección e intervención temprana y, además, en relación a un trabajo colaborativo entre diversos profesionales. Las evaluaciones neurológicas realizadas a la menor establecen que no hay alteraciones presentes, sin embargo, el retraso que la alumna presenta en el área del lenguaje y psicomotor sí es evidente y se puede asociar a los factores de riesgo a los que estuvo expuesta. Por tanto, es posible descartar el diagnóstico de TEL-Mixto, dadas las causas de origen neurobiológicas presentes en la menor. En base a esto, no sería un trastorno "específico" sino un trastorno del lenguaje asociado a una patología con un origen relacionado a la macrocefalia.

En la primera infancia debe existir un trabajo en equipo, tanto con los profesionales especializados en el área como también con la familia, siendo ésta el agente estimulador por excelencia, por lo cual es importante que participen y que se les pueda también ayudar a modificar o ajustar estrategias de interacción que desarrollen en sus casas con sus hijos para que el trabajo de atención temprana se considere completo, evitando así que los padres desde los primeros momentos desarrollen relaciones poco adecuadas con su hijo (Dunst, 2004; Giné et. Al, 2009; Sánchez y Candel, 2012).

Es relevante considerar, que los resultados del proceso de intervención solo muestran el avance inicial obtenido tras dos meses de terapia, por lo cual, es necesario una evaluación final tras un período de 6 o más meses de ejecutado el plan de trabajo con la menor y su contexto con el propósito de identificar de manera objetiva y a través de pruebas estandarizadas el nivel de avance obtenido.

Finalmente, se establece que la realización de intervenciones ajustadas, oportunas y acorde a las necesidades existentes pueden generar cambios beneficiosos para el desarrollo de una persona, más aún si la familia también 
se siente comprometida y capacitada para implementar estas estrategias en el hogar. Una de las aportaciones más importantes de este estudio de caso es el interés por mejorar las prácticas de intervención en lenguaje, psicomotoras y de funciones ejecutivas llevadas a cabo en los establecimientos educativos, partiendo de la base que deben ser identificadas en primera infancia con la finalidad de generar programas preventivos efectivos en los diferentes contextos en los cuales se desenvuelve un niño. En este sentido, la atención temprana cumple un rol esencial como procedimiento para dar respuesta lo más pronto posible a necesidades detectadas en niños durante su desarrollo o en aquellos que están en riesgo de padecerlas (Grupo de Atención Temprana, 2000).

\section{Agradecimientos}

Este trabajo fue desarrollado gracias al programa de Magíster en Educación Especial y Psicopedagogía de la Universidad Católica del Maule y al apoyo, y colaboración brindado por la Dra. María Teresa Muñoz Quezada en el curso de Neurociencia y Aprendizaje.

\section{REFERENCIAS BIBLIOGRÁFICAS}

Acosta, V., y Moreno, A. (1999). Las dificultades del lenguaje en ambientes educativos. Del retraso al trastorno específico del lenguaje. BarceIona: Masson.

Acosta, V., y Moreno, A. (2005). Dificultades del lenguaje en ambientes educativos, del retraso al trastorno específico del lenguaje. Barcelona, España: MASSON.

Acosta, V. (2012). Algunos retos y propuestas en la conceptualización, evaluación eintervención del Trastorno Específico del Lenguaje (TEL). Revista Chilena de Fonoaudiología, 17, 23-36, doi:10.5354/07194692.2012.24525

Allen, E., \& Cowdery, G. (2015). Facilitating speech, language, and communication skills from the exceptional child: inclusión in early childhood education. Cengage learning. 
American Psychiatric Association. (2013). Diagnostic and Statistical Manual of Mental Disorders (5a edition). Arlington: American Psychiatric Association.

Ardila, A. (2006). Orígenes del lenguaje: un análisis desde la perspectiva de las aphasias. Revista de Neurología, 43(11), 690-698.

Bloom, L., \& Lahey, M. (1978). Language development and language disorders. Nueva York: Wiley.

Burger, K. (2014). Effective early childhood care and education: Successful approaches and didactic strategies for fostering child development. European Early Childhood Education Research Journal, 8(2), 33-42. doi :10.1080/1350293X.2014.882076

Dunst, C. (2004). Revisiting, rethinking early intervention. EE.UU: Blackwell Publishing.

Feldman, R. (2007). Desarrollo Psicológico a través de la vida. (4ª ed.). México: Pearson.

Fundación Chile y Centro de Estudios MINEDUC. (2013). Análisis de la implementación de los programdas de ingtegración escolan (PIE) en establecimientos que han incorporado estudiantes con necesidades educativas especias/ transitorias. Recuperado de https://especial.mineduc.cl/wpcontent/uploads/sites/31/2016/08/Resumen_Estudio_ImplementaciónPIE_2013.pdf

García-Sánchez, F. A., Escorcia, C. T., Sánchez-López, M., Orcajada, N., y Hernández-Pérez, E. (2014). Atención temprana centrada en la familia. Siglo Cero: Revista Española Sobre Discapacidad Intelectual, 45(3), 6-27.

Giné, C., Gràcia, M., Vilaseca, R., y Balcells, A. (2009). Trabajar con las familias en atención temprana. Recuperado de http://feapscv.org/v2/wpcontent/uploads/Trabajar-con-Familias-en-AT.pdf

Grupo de Atención Temprana. (2000). Libro blanco de la atención temprana. Madrid: ARTEGRAF.

Haeussler, I., \& Marchant, T. (1985). Test de desarrollo psicomotor- TEPSI. Chile: Ediciones Universidad Católica de Chile. 
Levitt, P., Eagleson, KL., \& Powell, EM. (2004). Regulation of neocortical interneuron development and the implications for neurodevelopmental disorders. Trends Neurosci, 27(7), 400-406.

Ministerio de Educación. (2009). Decreto 170. Fija normas que determinan los alumnos con necesidades educativas especiales que serán beneficiados de las subvenciones para educación especial. Chile.

MINSAL. (2008). Manual para el apoyo y seguimiento del desarrollo psicosocial de los niños y niñas de 0 a 6 años. Recuperado de http://web. minsal.cl/sites/default/files/files/2008_Manual-para-el-Apoyo-y-Seguimiento-del-Desarrollo-Psicosocial-de-los-Ninos-y-Ninas-de-O-a6-Anos.pdf

Monfort, M., y Juárez, A. (2004). El niño que habla, el lenguaje oral en el preescolar. Madrid, España: CEPE.

Owens, R. (2008). Desarrollo del lenguaje (5 ed.). Madrid, España: Pearson Educación.

Puche, R., Orozco, M., Orozco, B., Correa, M., \& Corporación Niñez y Conocimiento. (2009). Desarrollo infantil y competencias en la primera infancia. Recuperado de http://www.mineducacion.gov.co/primerainfancia/1739/articles-178053_archivo_PDF_libro_desarrolloinfantil.pdf

Sánchez, J., y Candel, I. (2012). Aplicación de programas de atención temprana siguiendo un modelo educativo. Recuperado de http://www.scielo.br/ pdf/er/n43/n43aO4.pdf

Vigotsky, L. S. (1993). Pensamiento y lenguaje. Madrid: Aprendizaje Visor. 